\title{
A Functional Central Limit Theorem for Kernel Type Density Estimators
}

\author{
István Fazekas ${ }^{1}$ and Peter Filzmoser ${ }^{2}$ \\ ${ }^{1}$ University of Debrecen, Hungary \\ ${ }^{2}$ Vienna University of Technology, Austria
}

\begin{abstract}
Kernel type density estimators are studied for random fields. A functional central limit theorem in the space of square integrable functions is proved if the locations of observations become more and more dense in an increasing sequence of domains.
\end{abstract}

Zusammenfassung: Es werden Kerndichteschätzer für stochastische Felder untersucht. Ein funktionaler Grenzwertsatz im Raum der quadratisch integrierbaren Funktionen wird bewiesen für den Fall, dass die Lokationen der Beobachtungen immer dichter werden in einer wachsenden Folge von Bereichen.

Keywords: $L_{2}$-Space, Random Field, $\alpha$-Mixing, Infill Asymptotics, Increasing Domain Asymptotics.

\section{Introduction}

In this paper a functional central limit theorem in the space $L_{2}[0,1]$ is proved for kernel type density estimators for $\alpha$-mixing random fields if the locations of observations become more and more dense in an increasing sequence of domains.

Kernel type density estimators are widely studied, see e.g. Prakasa Rao (1983), Devroye and Györfi (1985). In Bosq et al. (1999), the asymptotic normality of these estimators is proved for $\alpha$-mixing stochastic sequences and continuous time processes. In Fazekas and Chuprunov (2005) a so called infill-increasing setup is used to obtain a result that is in some sense between the discrete and the continuous time cases. See also Fazekas and Chuprunov (2004).

In statistics, most asymptotic results concern the increasing domain case, i.e. when the random process (or field) is observed in an increasing sequence of domains $T_{n}$, with $\left|T_{n}\right| \rightarrow \infty$. However, if we observe a random field in a fixed domain and intend to prove an asymptotic theorem when the observations become dense in that domain, we obtain the so called infill asymptotics (see Cressie, 1991). It is known that several estimators being consistent for weakly dependent observations in the increasing domain setup are not consistent if the infill approach is considered.

In this paper we combine the infill and the increasing domain approaches. We call infill-increasing approach if our observations become more and more dense in an increasing sequence of domains. Using this setup, Lahiri (1999) and Fazekas (2003) studied the asymptotic behavior of the empirical distribution function. Also in the infill-increasing case, consistency and asymptotic normality of the least squares estimator for linear errorsin-variables models were proved in Fazekas and Kukush (2000). 
In this paper we follow the line of Fazekas and Chuprunov (2005). There asymptotic normality of the kernel type density estimator (4) is proved in the infill-increasing case. We quote that result in Theorem 2.1.

The main result of this paper is a functional central limit theorem for kernel type density estimators (Theorem 3.1). It is the functional version of the ordinary central limit theorem, i.e, Theorem 2.1.

We prove our functional central limit theorem in $L_{2}[0,1]$, i.e. in the space of square integrable functions defined in the interval $[0,1]$. We have to mention that most of the functional limit theorems are established in the space $C$ of continuous functions, or in the Skorohod space $D$, see Billingsley (1968). However, there are papers establishing criteria for functional limit theorems in $L_{p}$ and containing applications of such theorems (Grinblat, 1976, Ivanov, 1980, Oliveira and Suquet, 1998). To prove our result we apply criteria given in Grinblat (1976).

\section{Notation and a Central Limit Theorem}

The following notation is used. $\mathbb{Z}$ is the set of all integers, $\mathbb{Z}^{d}$ is the set of the $d$ dimensional lattice points, where $d$ is a fixed positive integer. $\mathbb{R}$ is the real line, $\mathbb{R}^{d}$ is the $d$-dimensional space with the usual Euclidean norm $\|\mathbf{x}\|$. In $\mathbb{R}^{d}$ we shall also consider the distance corresponding to the maximum norm: $\varrho(\mathbf{x}, \mathbf{y})=\max _{1 \leq i \leq d}\left|x^{(i)}-y^{(i)}\right|$, where $\mathbf{x}=\left(x^{(1)}, \ldots, x^{(d)}\right), \mathbf{y}=\left(y^{(1)}, \ldots, y^{(d)}\right)$. The distance of two sets in $\mathbb{R}^{d}$ corresponding to the maximum norm is also denoted by $\varrho$, i.e. $\varrho(A, B)=\inf \{\varrho(a, b): a \in A, b \in B\}$.

We shall denote different finite constants with the same letter $C . \quad|\mathcal{D}|$ denotes the cardinality of the finite set $\mathcal{D}$ and at the same time $|T|$ denotes the volume of the domain $T$. The indicator function of the set $A$ is $\mathrm{I}\{A\}$.

We shall suppose the existence of an underlying probability space $(\Omega, \mathcal{F}, \mathbb{P})$. The $\sigma$ algebra generated by a set of events or by a set of random variables will be denoted by $\sigma\{$.$\} . The L_{p}$-norm of a random (vector) variable $\eta$ is $\|\eta\|_{p}=\left\{\mathbb{E}\|\eta\|^{p}\right\}^{1 / p}, 1 \leq p<\infty$.

Sign $\Rightarrow$ denotes convergence in distribution. $\mathcal{N}(m, \Sigma)$ stands for the (vector) normal distribution with mean (vector) $m$ and covariance (matrix) $\Sigma$.

Describe the scheme of observations. For simplicity we restrict ourselves to rectangles as domains of observations. Let $\Lambda>0$ be fixed. By $(\mathbb{Z} / \Lambda)^{d}$ we denote the $\Lambda$-lattice points in $\mathbb{R}^{d}$, i.e. lattice points with distance $1 / \Lambda$ :

$$
\left(\frac{\mathbb{Z}}{\Lambda}\right)^{d}=\left\{\left(\frac{k_{1}}{\Lambda}, \ldots, \frac{k_{d}}{\Lambda}\right):\left(k_{1}, \ldots, k_{d}\right) \in \mathbb{Z}^{d}\right\} .
$$

$T$ will be a bounded, closed rectangle in $\mathbb{R}^{d}$ with edges parallel to the axes and $\mathcal{D}$ will denote the $\Lambda$-lattice points belonging to $T$, i.e. $\mathcal{D}=T \cap(\mathbb{Z} / \Lambda)^{d}$. To describe the limit distribution we consider a sequence of the previous objects. I.e. let $T_{1}, T_{2}, \ldots$ be bounded, closed rectangles in $\mathbb{R}^{d}$. Suppose that

$$
T_{1} \subset T_{2} \subset T_{3} \subset \cdots, \quad \bigcup_{i=1}^{\infty} T_{i}=T_{\infty} .
$$


We assume that the length of each edge of $T_{n}$ is integer and converges to $\infty$, as $n \rightarrow \infty$. Let $\left\{\Lambda_{n}\right\}$ be an increasing sequence of positive integers (the non-integer case is essentially the same) and $\mathcal{D}_{n}$ be the $\Lambda_{n}$-lattice points belonging to $T_{n}$.

Let $\left\{\xi_{\mathbf{t}}, \mathbf{t} \in T_{\infty}\right\}$ be a random field. The $n$-th set of observations involves the values of the random field $\xi_{\mathbf{t}}$ taken at each point $\mathbf{k} \in \mathcal{D}_{n}$. Actually, each $\mathbf{k}=\mathbf{k}^{(n)} \in \mathcal{D}_{n}$ depends on $n$ but to avoid complicated notation we often omit the superscript $(n)$. By our assumptions, $\lim _{n \rightarrow \infty}\left|\mathcal{D}_{n}\right|=\infty$.

We need the notion of $\alpha$-mixing (see e.g. Doukhan, 1994, Guyon, 1995, Lin and Lu, 1996). Let $\mathcal{A}$ and $\mathcal{B}$ be two $\sigma$-algebras in $\mathcal{F}$. The $\alpha$-mixing coefficient of $\mathcal{A}$ and $\mathcal{B}$ is defined as follows:

$$
\alpha(\mathcal{A}, \mathcal{B})=\sup \{|\mathbb{P}(A) \mathbb{P}(B)-\mathbb{P}(A B)|: A \in \mathcal{A}, B \in \mathcal{B}\} .
$$

The $\alpha$-mixing coefficients of $\left\{\xi_{\mathbf{t}}: \mathbf{t} \in T_{\infty}\right\}$ are

$$
\begin{gathered}
\alpha(r, u, v)=\sup \left\{\alpha\left(\mathcal{F}_{I_{1}}, \mathcal{F}_{I_{2}}\right): \varrho\left(I_{1}, I_{2}\right) \geq r,\left|I_{1}\right| \leq u,\left|I_{2}\right| \leq v\right\}, \\
\alpha(r)=\sup \left\{\alpha\left(\mathcal{F}_{I_{1}}, \mathcal{F}_{I_{2}}\right): \varrho\left(I_{1}, I_{2}\right) \geq r\right\},
\end{gathered}
$$

where $I_{i}$ is a finite subset in $T_{\infty}$ with cardinality $\left|I_{i}\right|$ and $\mathcal{F}_{I_{i}}=\sigma\left\{\xi_{\mathbf{t}}: \mathbf{t} \in I_{i}\right\}, i=1,2$. We shall use the following condition. For some $1<a<\infty$

$$
\int_{0}^{\infty} s^{2 d-1} \alpha^{\frac{a-1}{a}}(s) d s<\infty .
$$

From now on we shall use the following assumptions throughout the paper.

Suppose that $\xi_{\mathbf{t}}, \mathbf{t} \in T_{\infty}$, is a strictly stationary random field with unknown continuous marginal density function $f$. We shall estimate $f$ from the data $\xi_{\mathbf{i}}, \mathbf{i} \in \mathcal{D}_{n}$.

A function $K: \mathbb{R} \rightarrow \mathbb{R}$ will be called a kernel if $K$ is a bounded, continuous, symmetric density function (with respect to the Lebesgue measure), and

$$
\lim _{|u| \rightarrow \infty}|u| K(u)=0, \quad \int_{-\infty}^{+\infty} u^{2} K(u) d u<\infty .
$$

Let $K$ be a kernel and let $h_{n}>0$, then the kernel-type density estimator is

$$
f_{n}(x)=\frac{1}{\left|\mathcal{D}_{n}\right|} \sum_{\mathbf{i} \in \mathcal{D}_{n}} \frac{1}{h_{n}} K\left(\frac{x-\xi_{\mathbf{i}}}{h_{n}}\right), \quad x \in \mathbb{R} .
$$

Let $f_{\mathbf{u}}(x, y)$ be the joint density function of $\xi_{\mathbf{0}}$ and $\xi_{\mathbf{u}}$, if $\mathbf{u} \neq \mathbf{0}$. Denote $\mathbb{R}_{\mathbf{0}}^{d}$ the set $\mathbb{R}^{d} \backslash\{\mathbf{0}\}$. Let

$$
g_{\mathbf{u}}(x, y)=f_{\mathbf{u}}(x, y)-f(x) f(y), \quad \mathbf{u} \in \mathbb{R}_{\mathbf{0}}^{d}, x, y \in \mathbb{R} .
$$

We assume that $g_{\mathbf{u}}(x, y)$ is continuous in $x$ and $y$ for each fixed $\mathbf{u}$.

Denote by $C\left(\mathbb{R}^{2}\right)$ the space of continuous real-valued functions over $\mathbb{R}^{2}$. Consider the function $\mathbf{u} \rightarrow g_{\mathbf{u}}(\cdot, \cdot), \mathbf{u} \in \mathbb{R}_{\mathbf{0}}^{d}$. This is an $\mathbb{R}_{\mathbf{0}}^{d} \rightarrow C\left(\mathbb{R}^{2}\right)$ mapping. For the sake of brevity we denote this function by $g_{\mathbf{u}}$ (and we consider it as the above $\mathbb{R}_{\mathbf{0}}^{d} \rightarrow C\left(\mathbb{R}^{2}\right)$ mapping). Let $\left\|g_{\mathbf{u}}\right\|=\sup _{(x, y) \in \mathbb{R}^{2}}\left|g_{\mathbf{u}}(x, y)\right|$. It is the norm of $g_{\mathbf{u}}(\cdot, \cdot)$ in $C\left(\mathbb{R}^{2}\right)$. In order to keep 
the notation simple, the function $\mathbf{u} \rightarrow\left\|g_{\mathbf{u}}\right\|$ (which is an $\mathbb{R}_{\mathbf{0}}^{d} \rightarrow \mathbb{R}$ mapping) will also be denoted by $\left\|g_{\mathbf{u}}\right\|$.

Introduce the notation

$$
\sigma(x, y)=\int_{\mathbb{R}_{\mathbf{0}}^{d}} g_{\mathbf{u}}(x, y) d \mathbf{u}, \quad x, y \in \mathbb{R} .
$$

For a fixed positive integer $m$ and fixed distinct real numbers $x_{1}, \ldots, x_{m}$, let

$$
\Sigma^{(m)}=\left(\sigma\left(x_{i}, x_{j}\right)\right)_{1 \leq i, j \leq m} .
$$

Theorem 2.1. (Theorem 1 in Fazekas and Chuprunov, 2005.) Assume that $g_{\mathbf{u}}$ is Riemann integrable (as a function $\mathbb{R}_{0}^{d} \rightarrow C\left(\mathbb{R}^{2}\right)$ ) on each bounded closed d-dimensional rectangle $R \subset \mathbb{R}_{0}^{d}$, moreover $\left\|g_{\mathbf{u}}\right\|$ is directly Riemann integrable (as a function $\mathbb{R}_{0}^{d} \rightarrow \mathbb{R}$ ). Let $x_{1}, \ldots, x_{m}$ be given distinct real numbers and assume that $\Sigma^{(m)}$ in (7) is positive definite. Suppose that there exists $1<a<\infty$ such that (2) is satisfied and

$$
\left(h_{n}\right)^{-1} \leq C\left|T_{n}\right|^{\frac{a^{2}}{(3 a-1)(2 a-1)}} \text { for each } n .
$$

Assume that $\lim _{n \rightarrow \infty} \Lambda_{n}=\infty, \lim _{n \rightarrow \infty} h_{n}=0$, and

$$
\lim _{n \rightarrow \infty} \frac{1}{\Lambda_{n}^{d}} \frac{1}{h_{n}}=L
$$

Then

$$
\sqrt{\frac{\left|\mathcal{D}_{n}\right|}{\Lambda_{n}^{d}}}\left\{\left(f_{n}\left(x_{i}\right)-\mathbb{E} f_{n}\left(x_{i}\right)\right), i=1, \ldots, m\right\} \Rightarrow \mathcal{N}\left(0, \Sigma^{\prime(m)}\right),
$$

as $n \rightarrow \infty$, where

$$
\Sigma^{\prime(m)}=\Sigma^{(m)}+D,
$$

and $D$ is a diagonal matrix with diagonal elements $L f\left(x_{i}\right) \int_{-\infty}^{+\infty} K^{2}(u) d u, i=1, \ldots, m$.

If $f(x)$ has bounded second derivative and $\lim _{n \rightarrow \infty}\left|T_{n}\right| h_{n}^{4}=0$, then in (10) $\mathbb{E} f_{n}\left(x_{i}\right)$ can be changed for $f\left(x_{i}\right), i=1, \ldots, m$, and the above statement remains valid.

Remark 2.2. In Theorem 2.1 and Theorem 3.1 we need approximations of the integral $\int_{\mathbb{R}_{0}^{d}}\left\|g_{\mathbf{u}}\right\| d \mathbf{u}$ with Riemannian sums. This procedure is usually applied only for bounded functions defined in bounded closed domains. Therefore we turn to the idea of direct Riemann integrability. The notion of direct Riemann integrability is well-known for univariate functions (see, e.g., Asmussen, 1987, p. 118). That is somewhat stronger than Lebesgue integrability. As we did not find appropriate references for multivariate functions, below we describe the notion of direct Riemann integrability for nonnegative functions defined on $\mathbb{R}_{0}^{d}$ and being unbounded at the origin.

Let $l: \mathbb{R}_{0}^{d} \rightarrow[0, \infty)$ be given. For a $v>0$ consider a subdivision of $\mathbb{R}^{d}$ into (right closed and left open) $d$-dimensional cubes $\Delta_{\mathbf{i}}$ with edge length $v$ such that the center of $\Delta_{\mathbf{0}}$ is the origin $\mathbf{0} \in \mathbb{R}^{d}$. The family $\left\{\Delta_{\mathrm{i}}\right\}$ is called the subdivision corresponding to $v$. If $\mathbf{i} \neq \mathbf{0}$, for $\mathbf{x} \in \Delta_{\mathbf{i}}$ let $\bar{l}_{v}(\mathbf{x})=\sup \left\{l(\mathbf{y}): \mathbf{y} \in \Delta_{\mathbf{i}}\right\}, \underline{l}_{v}(\mathbf{x})=\inf \left\{l(\mathbf{y}): \mathbf{y} \in \Delta_{\mathbf{i}}\right\}$, while $\bar{l}_{v}(\mathbf{x})=\underline{l}_{v}(\mathbf{x})=0$ if $\mathbf{x} \in \Delta_{0}$. If

$$
\lim _{v \rightarrow 0} \int_{\mathbb{R}^{d}} \bar{l}_{v}(\mathbf{x}) d \mathbf{x}=\lim _{v \rightarrow 0} \int_{\mathbb{R}^{d}} \underline{l}_{v}(\mathbf{x}) d \mathbf{x}=I
$$


and this common value is finite, then $l$ is called directly Riemann integrable (d.R.i.) and $I$ is its direct Riemann integral.

If $l$ is d.R.i., then $l$ is bounded outside each neighborhood of the origin. Moreover, $l$ is continuous almost everywhere (with respect to the Lebesgue measure). Therefore, by the Lebesgue criterion of Riemann integrability (see Zorich, 1984), $l$ is Riemann integrable on each bounded closed $d$-dimensional rectangle not containing the origin. Call a zone a set $M=R_{1} \backslash R_{2}$, where $R_{1}$ is a closed $d$-dimensional rectangle while $R_{2}\left(\emptyset \neq R_{2} \subset R_{1}\right)$ is an open $d$-dimensional rectangle both having center at the origin. Then one obtains that $l$ is Riemann integrable on each zone.

If $l \geq 0$ is d.R.i. then the improper integral $\int_{\mathbb{R}_{0}^{d}} l(\mathbf{x}) d \mathbf{x}$ exists and it is equal to the direct Riemann integral of $l$. This can be proved using zones approximating $\mathbb{R}_{0}^{d}$ and the properties of the improper integral of a nonnegative function (see Zorich, 1984). The above statement implies: for any $\varepsilon>0$ there exists a zone $M$ such that $\int_{\mathbb{R}_{\mathbf{0}}^{d} \backslash M} l(\mathbf{x}) d \mathbf{x} \leq \varepsilon$.

Finally, we have the following. Let $l \geq 0$ be d.R.i. Let $v_{n}$ be positive numbers converging to zero, and let $\left\{\Delta_{\mathrm{i}}^{(n)}\right\}$ be the subdivision corresponding to $v_{n}$. Then for any $\varepsilon>0$ there exists a zone $M$ such that all Riemannian approximating sums (based on the above subdivisions but not containing term $\left.\left|\Delta_{\mathbf{0}}\right| l\left(\mathbf{x}_{\mathbf{0}}\right)\right)$ of the integral $\int_{\mathbb{R}_{\mathbf{0}}^{d} \backslash M} l(\mathbf{x}) d \mathbf{x}$ are less than $\varepsilon$.

For the definition of the Riemann integrability of a Banach space valued function, see Hille and Phillips (1957, p. 62).

\section{The Functional Central Limit Theorem}

In this section we shall prove a functional central limit theorem in the space $L_{2}[0,1]$. We shall use the assumptions of the previous section. Moreover, in this section we suppose that both $f$ and $f_{n}$ are equal to 0 outside of the interval $[0,1]$. If we restrict our study to densities and kernel functions with compact supports, by appropriate transformation, this condition can be realized.

Let

$$
L_{n}(x)=\sqrt{\frac{\left|\mathcal{D}_{n}\right|}{\Lambda_{n}^{d}}}\left[f_{n}(x)-f(x)\right] .
$$

In the following theorem we shall use conditions of Theorem 2.1.

Theorem 3.1. Assume that $g_{\mathbf{u}}$ is Riemann integrable on each bounded closed $d$ dimensional rectangle $R \subset \mathbb{R}_{0}^{d}$, moreover $\left\|g_{\mathbf{u}}\right\|$ is directly Riemann integrable. Let the function $\sigma(x, y)$ defined in (6) be positive definite. Suppose that there exists $1<a<\infty$ such that (2) and (8) are satisfied. Assume that $\lim _{n \rightarrow \infty} \Lambda_{n}=\infty, \lim _{n \rightarrow \infty} h_{n}=0$, and

$$
\lim _{n \rightarrow \infty} \frac{1}{\Lambda_{n}^{d}} \frac{1}{h_{n}}=0
$$

Assume that $f(x)$ has bounded second derivative and $\lim _{n \rightarrow \infty}\left|T_{n}\right| h_{n}^{4}=0$.

Then, as $n \rightarrow \infty$,

$$
L_{n}(x) \Rightarrow G(x)
$$


in $L_{2}[0,1]$, where $G$ is a Gaussian process with mean 0 and with covariance function $\sigma(\cdot, \cdot)$.

To prove our theorem we need the following criterion (see Grinblat, 1976, and Ivanov, 1980).

Proposition. Let $\xi(t)$ and $\xi_{n}(t), n=1,2, \ldots$, be measurable stochastic processes on $[0,1]$. Assume

the finite dimensional distributions of $\xi_{n}(t)$ converge to those of $\xi(t)$;

$$
\begin{gathered}
\sup _{n} \sup _{t} \mathbb{E}\left(\xi_{n}(t)\right)^{2}=c<\infty ; \\
\lim _{n \rightarrow \infty} \mathbb{E}\left(\xi_{n}(t)\right)^{2}=\mathbb{E}(\xi(t))^{2} \quad \text { for each } t \in[0,1] .
\end{gathered}
$$

Then, as $n \rightarrow \infty, \xi_{n}(t) \Rightarrow \xi(t)$ in $L_{2}[0,1]$.

Proof of Theorem 3.1. First we prove that

$$
L_{n}^{0}(x) \Rightarrow G(x)
$$

in $L_{2}[0,1]$, where

$$
\begin{aligned}
L_{n}^{0}(x) & =\sqrt{\frac{\left|\mathcal{D}_{n}\right|}{\Lambda_{n}^{d}}}\left[f_{n}(x)-\mathbb{E} f_{n}(x)\right] \\
& =\frac{1}{\sqrt{\left|\mathcal{D}_{n}\right| \Lambda_{n}^{d}}} \sum_{\mathbf{i} \in \mathcal{D}_{n}}\left\{\frac{1}{h_{n}} K\left(\frac{x-\xi_{\mathbf{i}}}{h_{n}}\right)-\frac{1}{h_{n}} \mathbb{E} K\left(\frac{x-\xi_{\mathbf{i}}}{h_{n}}\right)\right\} .
\end{aligned}
$$

To this end we have to check the conditions of the preceding proposition.

Condition (15), i.e. the convergence of the finite dimensional distributions of $L_{n}^{0}(x)$ to those of $G(x)$ is a consequence of Theorem 2.1.

Now we turn to (16). The following calculation is a version of what is included in the proof of Theorem 1 in Fazekas and Chuprunov (2005).

$$
\begin{aligned}
\mathbb{E}\left(L_{n}^{0}(x)\right)^{2} & =\frac{1}{\Lambda_{n}^{d}\left|\mathcal{D}_{n}\right|} \sum_{\mathbf{i} \in \mathcal{D}_{n}} \sum_{\mathbf{j} \in \mathcal{D}_{n}} \operatorname{cov}\left\{\frac{1}{h_{n}} K\left(\frac{x-\xi_{\mathbf{i}}}{h_{n}}\right), \frac{1}{h_{n}} K\left(\frac{x-\xi_{\mathbf{j}}}{h_{n}}\right)\right\} \\
& =A_{n}(x)+B_{n}(x),
\end{aligned}
$$

where $A_{n}(x)$ denotes the part of the sum with $\mathbf{i}=\mathbf{j}$, while $B_{n}(x)$ denotes the part of the sum with $\mathbf{i} \neq \mathbf{j}$.

For $A_{n}(x)$ we have

$$
\begin{aligned}
A_{n}(x) & =\frac{1}{\Lambda_{n}^{d} h_{n}} \operatorname{var}\left\{\frac{1}{\sqrt{h_{n}}} K\left(\frac{x-\xi_{\mathbf{i}}}{h_{n}}\right)\right\} \\
& =\frac{1}{\Lambda_{n}^{d} h_{n}}\left\{\int_{-\infty}^{+\infty} \frac{1}{h_{n}} K^{2}\left(\frac{x-u}{h_{n}}\right) f(u) d u-h_{n}\left[\int_{-\infty}^{+\infty} \frac{1}{h_{n}} K\left(\frac{x-u}{h_{n}}\right) f(u) d u\right]^{2}\right\} \\
& =\frac{1}{\Lambda_{n}^{d} h_{n}}\left\{\int_{-\infty}^{+\infty} K^{2}(y) f\left(x-h_{n} y\right) d y-h_{n}\left[\int_{-\infty}^{+\infty} K(y) f\left(x-h_{n} y\right) d y\right]^{2}\right\} \\
& \leq \tilde{L}\left\{C \int_{-\infty}^{+\infty} K^{2}(y) d y-h_{n} C^{2}\left[\int_{-\infty}^{+\infty} K(y) d y\right]^{2}\right\}<\infty,
\end{aligned}
$$


as $f$ (being continuous on a compact set) is bounded.

Now, turn to $B_{n}(x)$.

$$
B_{n}(x)=\frac{1}{\Lambda_{n}^{d}\left|\mathcal{D}_{n}\right|} \sum \sum_{\mathbf{i} \neq \mathbf{j}} \int_{-\infty}^{+\infty} \int_{-\infty}^{+\infty} \frac{1}{h_{n}} K\left(\frac{x-u}{h_{n}}\right) \frac{1}{h_{n}} K\left(\frac{x-v}{h_{n}}\right) g_{\mathbf{i}-\mathbf{j}}(u, v) d u d v
$$

As the random field is strictly stationary, we can assume that the center of the rectangle $T_{n}$ is the origin. Then the set of vectors of the form $\mathbf{i}-\mathbf{j}$ with $\mathbf{i}, \mathbf{j} \in \mathcal{D}_{n}$ is $2 \mathcal{D}_{n}$, where $2 \mathcal{D}_{n}$ is defined as $\left(2 T_{n}\right) \cap\left(\mathbb{Z} / \Lambda_{n}\right)^{d}$. If $\mathbf{u} \in 2 \mathcal{D}_{n}$ is fixed, then denote by $\left|\mathcal{D}_{n, \mathbf{u}}\right|$ the number of pairs $(\mathbf{i}, \mathbf{j}) \in \mathcal{D}_{n} \times \mathcal{D}_{n}$ with $\mathbf{i}-\mathbf{j}=\mathbf{u}$. Then

$$
B_{n}(x)=\int_{-\infty}^{+\infty} \int_{-\infty}^{+\infty}\left\{\frac{1}{h_{n}} K\left(\frac{x-u}{h_{n}}\right) \frac{1}{h_{n}} K\left(\frac{x-v}{h_{n}}\right)\left(\frac{1}{\Lambda_{n}^{d}} \sum_{\mathbf{u} \in 2 \mathcal{D}_{n}^{0}} \frac{\left|\mathcal{D}_{n, \mathbf{u}}\right|}{\left|\mathcal{D}_{n}\right|} g_{\mathbf{u}}(u, v)\right)\right\} d u d v
$$

where $2 \mathcal{D}_{n}^{0}=2 \mathcal{D}_{n} \backslash\{\boldsymbol{0}\}$. Now fix an $\varepsilon>0$. As $\left\|g_{\mathbf{u}}\right\|$ is directly Riemann integrable, one can find a zone $M_{\varepsilon} \subset \mathbb{R}^{d}$ (with center in the origin) such that

$$
\int_{\mathbb{R}_{\mathbf{0}}^{d} \backslash M_{\varepsilon}}\left\|g_{\mathbf{u}}\right\| d \mathbf{u} \leq \varepsilon
$$

and at the same time the Riemannian approximating sums of this integral do not exceed $\varepsilon$ if the diameter of the subdivision is small enough. Therefore, as $\left|\mathcal{D}_{n, \mathbf{u}}\right| /\left|\mathcal{D}_{n}\right| \leq 1$,

$$
\frac{1}{\Lambda_{n}^{d}} \sum_{\mathbf{u} \in\left\{2 \mathcal{D}_{n}^{0} \backslash M_{\varepsilon}\right\}} \frac{\left|\mathcal{D}_{n, \mathbf{u}}\right|}{\left|\mathcal{D}_{n}\right|}\left\|g_{\mathbf{u}}\right\| \leq \varepsilon
$$

when $1 / \Lambda_{n}^{d}$ is small enough, i.e. when $n$ is large enough: $n \geq n_{\varepsilon}$. Fix $\varepsilon, M_{\varepsilon}$ and assume that $n \geq n_{\varepsilon}$. Because $g_{\mathbf{u}}$ is Riemann integrable as a function $\mathbb{R}_{0}^{d} \rightarrow C\left(\mathbb{R}^{2}\right)$ on $R$ for each bounded closed $d$-dimensional rectangle $R$ in $\mathbb{R}_{0}^{d}$, therefore we have

$$
\left\|\frac{1}{\Lambda_{n}^{d}} \sum_{\mathbf{u} \in 2 \mathcal{D}_{n}^{0} \cap M_{\varepsilon}} g_{\mathbf{u}}-\int_{M_{\varepsilon}} g_{\mathbf{u}} d \mathbf{u}\right\| \leq \varepsilon
$$

in the space $C\left(\mathbb{R}^{2}\right)$, if $n$ is large enough. This relation and (22) imply that $\int_{\mathbb{R}_{0}^{d}} g_{\mathbf{u}}(x, y) d \mathbf{u}$ exists and it is continuous in $(x, y)$. As each edge of $T_{n}$ converges to $\infty,\left|\mathcal{D}_{n, \mathbf{u}}\right| /\left|\mathcal{D}_{n}\right| \rightarrow 1$ uniformly according to $\mathbf{u} \in M_{\varepsilon}$. Therefore, using that $\left\|g_{\mathbf{u}}\right\|$ is directly Riemann integrable, we obtain that

$$
\left\|\frac{1}{\Lambda_{n}^{d}} \sum_{\mathbf{u} \in 2 \mathcal{D}_{n}^{\mathbf{0}} \cap M_{\varepsilon}} \frac{\left|\mathcal{D}_{n, \mathbf{u}}\right|}{\left|\mathcal{D}_{n}\right|} g_{\mathbf{u}}-\frac{1}{\Lambda_{n}^{d}} \sum_{\mathbf{u} \in 2 \mathcal{D}_{n}^{\mathbf{0}} \cap M_{\varepsilon}} g_{\mathbf{u}}\right\| \leq \varepsilon,
$$

if $n$ is large enough.

Relations (22), (23), (24), and (25) imply that

$$
\left\|\frac{1}{\Lambda_{n}^{d}} \sum_{\mathbf{u} \in 2 \mathcal{D}_{n}^{0}} \frac{\left|\mathcal{D}_{n, \mathbf{u}}\right|}{\left|\mathcal{D}_{n}\right|} g_{\mathbf{u}}-\int_{\mathbb{R}_{\mathbf{0}}^{d}} g_{\mathbf{u}} d \mathbf{u}\right\| \leq 4 \varepsilon
$$


if $n$ is large enough.

Therefore, using that $\frac{1}{h_{n}} K\left(\frac{x-u}{h_{n}}\right)$ is a density function, we have

$$
\left|B_{n}(x)-\int_{-\infty}^{+\infty} \int_{-\infty}^{+\infty}\left\{\frac{1}{h_{n}} K\left(\frac{x-u}{h_{n}}\right) \frac{1}{h_{n}} K\left(\frac{x-v}{h_{n}}\right) \int_{\mathbb{R}_{\mathbf{0}}^{d}} g_{\mathbf{u}}(u, v) d \mathbf{u}\right\} d u d v\right| \leq 4 \varepsilon
$$

if $n$ is large enough. Moreover, the second term between the above absolute value signs is equal to

$$
\int_{-\infty}^{+\infty} \int_{-\infty}^{+\infty} K(t) K(s)\left\{\int_{\mathbb{R}_{\mathbf{0}}^{d}} g_{\mathbf{u}}\left(x-h_{n} t, x-h_{n} s\right) d \mathbf{u}\right\} d t d s
$$

As $\left\|g_{\mathbf{u}}\right\|$ is directly Riemann integrable, and $K$ is a density function, therefore the above expression is bounded. So condition (16) is satisfied.

The proof of condition (17), i.e. $\lim _{n \rightarrow \infty} \mathbb{E}\left(L_{n}^{0}(x)\right)^{2}=\mathbb{E}(G(x))^{2}$, for each $x \in[0,1]$ is included in the proof of Theorem 1 in Fazekas and Chuprunov (2005), as there the convergences of the covariances are checked.

Now, to prove (14), we have to consider the difference

$$
\begin{aligned}
L_{n}(x)-L_{n}^{0}(x) & =\sqrt{\frac{\left|\mathcal{D}_{n}\right|}{\Lambda_{n}^{d}}}\left[\mathbb{E} f_{n}(x)-f(x)\right] \\
& =\frac{\mathbb{E} f_{n}(x)-f(x)}{h_{n}^{2}} \sqrt{\frac{\left|\mathcal{D}_{n}\right|}{\Lambda_{n}^{d}}} h_{n}^{2} .
\end{aligned}
$$

Here $h_{n}^{2} \sqrt{\left|\mathcal{D}_{n}\right| / \Lambda_{n}^{d}}=h_{n}^{2} \sqrt{\left|T_{n}\right|} \rightarrow 0$. On the other hand, using Taylor's expansion,

$$
\begin{aligned}
\mathbb{E} f_{n}(x)-f(x) & =\int_{-\infty}^{+\infty} K(t) f\left(x-h_{n} t\right) d t-f(x) \\
=f(x) \int_{-\infty}^{+\infty} K(t) d t & -f^{\prime}(x) h_{n} \int_{-\infty}^{+\infty} t K(t) d t+\frac{h_{n}^{2}}{2} \int_{-\infty}^{+\infty} t^{2} K(t) f^{\prime \prime}\left(\vartheta\left(h_{n} t, x\right)\right) d t-f(x) \\
& =\frac{h_{n}^{2}}{2} \int_{-\infty}^{+\infty} t^{2} K(t) f^{\prime \prime}\left(\vartheta\left(h_{n} t, x\right)\right) d t
\end{aligned}
$$

where $\vartheta\left(h_{n} t, x\right)$ is a point between $x$ and $x-h_{n} t$. This expression converges to 0 , uniformly in $x$, because $h_{n} \rightarrow 0, f^{\prime \prime}$ is bounded, and $\int_{-\infty}^{+\infty} t^{2} K(t) d t<\infty$. So we obtain that

$$
L_{n}(x)-L_{n}^{0}(x) \rightarrow 0
$$

uniformly in $x$. 


\section{Acknowledgements}

Research of the first author was supported by the Hungarian Foundation of Scientific Researches under Grant No. OTKA T047067/2004 and Grant No. OTKA T048544/2005. The authors are indebted to the referee whose suggestions helped to improve the presentation of the results.

\section{References}

Asmussen, S. (1987). Applied Probability and Queues. New York: Wiley.

Billingsley, P. (1968). Convergence of Probability Measures. New York: Wiley.

Bosq, D., Merlevède, F., and Peligrad, M. (1999). Asymptotic normality for density kernel estimators in discrete and continuous time. Journal of Multivariate Analysis, 68, 78-95.

Cressie, N. A. C. (1991). Statistics for Spatial Data. New York: Wiley.

Devroye, L., and Györfi, L. (1985). Nonparametric Density Estimation. The $L_{1}$ View. New York: Wiley.

Doukhan, P. (1994). Mixing. Properties and Examples (Vols. 85, Lecture Notes in Statistics). New York: Springer.

Fazekas, I. (2003). Limit theorems for the empirical distribution function in the spatial case. Statistics \& Probability Letters, 62, 251-262.

Fazekas, I., and Chuprunov, A. (2004). A central limit theorem for random fields. Acta Mathematica Academiae Paedagogicae Nyíregyháziensis, 20(1), 93-104.

Fazekas, I., and Chuprunov, A. (2005). Asymptotic normality of kernel type density estimators for random fields. Accepted by Statistical Inference for Stochastic Processes.

Fazekas, I., and Kukush, A. G. (2000). Infill asymptotics inside increasing domains for the least squares estimator in linear models. Statistical Inference for Stochastic Processes, 3, 199-223.

Grinblat, L. S. (1976). A limit theorem for measurable random processes and its applications. Proceedings of the American Mathematical Society, 61, 371-376.

Guyon, X. (1995). Random Fields on a Network. Modeling, Statistics, and Applications. New York: Springer.

Hille, E., and Phillips, R. S. (1957). Functional Analysis and Semi-Groups. Providence: American Mathematical Society.

Ivanov, A. V. (1980). On convergence of distributions of functionals of measurable random fields. Ukrainean Journal of Mathematics, 32(1), 27-34.

Lahiri, S. N. (1999). Asymptotic distribution of the empirical spatial cumulative distribution function predictor and prediction bands based on a subsampling method. Probability Theory and Related Fields, 114, 55-84.

Lin, Z., and Lu, C. (1996). Limit Theory for Mixing Dependent Random Variables. Dordrecht: Kluwer Academic Publishers.

Oliveira, P. E., and Suquet, C. (1998). Weak convergence in $L_{p}(0,1)$ of the uniform empirical process under dependence. Statistics \& Probability Letters, 39(4), 363370 . 
Prakasa Rao, B. L. S. (1983). Nonparametric Functional Estimation. New York: Academic Press.

Zorich, V. A. (1984). Mathematical Analysis. (In Russian.) (Vol. II). Moscow: Nauka.

Authors' addresses:

István Fazekas

Faculty of Informatics

University of Debrecen

P.O. Box 12

H-4010 Debrecen, Hungary

E-mail: fazekasi@inf.unideb.hu

Peter Filzmoser

Department of Statistics and Probability Theory

Vienna University of Technology

Wiedner Hauptstraße 8-10

A-1040 Vienna, Austria

E-mail: P.Filzmoser@tuwien.ac.at 